\title{
Modeling aging rates in a simple glass and its melt
}

\author{
H. R. Schober \\ Institut für Festkörperforschung, Forschungszentrum Jülich, D-52425 Jülich, Germany
}

(Received 20 December 2011; published 12 January 2012)

\begin{abstract}
We calculated with a molecular dynamics simulation the aging rates in a binary Lennard-Jones glass and its undercooled melt. At temperatures above the mode coupling theory (MCT) critical temperature $T_{c}$, pressure or volume, internal energy, and diffusivity age with the same rate. Below $T_{c}$ we see a split of the aging rates into a fast one for the diffusivity and a much slower one for pressure or volume and internal energy. The latter aging rate is roughly proportional to the diffusivity. The observed stretched exponential behavior is shown to stem from the faster aging of the diffusivity. Aging of of internal energy and pressure proceeds exponentially with the mean-square displacement. The exponential prefactor exhibits the kink at $T_{c}$ seen earlier in the pressure dependence of the diffusivity.
\end{abstract}

DOI: 10.1103/PhysRevB.85.024204

PACS number(s): 61.43.Fs, 64.70.pe

\section{INTRODUCTION}

If a liquid is quenched to a lower temperature or different pressure it takes time to relax to its thermodynamical equilibrium state. The lower the temperature, the longer the relaxation time. Crossing the glass transition temperature $T_{g}$, thermodynamic equilibrium will no longer be reached in experimental times. The liquid state has changed to the glassy one. The relaxation toward thermodynamic equilibrium - the aging - affects most properties of the glass and is, therefore, of great technological importance and has been studied widely. Most often the decrease of enthalpy or volume with time is followed. ${ }^{1}$ The relaxation toward equilibrium slows down with time, and is commonly described by a Kohlrausch-WilliamsWatts (KWW) stretched exponential expression, sometimes with additional modifications. The physical origin of the stretching is still disputed. The time evolution of the dynamics in simple glass formers has been studied less frequently. One line of investigation is the study of the frequency-dependent dielectric susceptibility, e.g., in glycerol, ${ }^{2,3}$ which again ages with a KWW law. Metallic glasses represent the most simple systems with no internal freedom. Their dynamics is restricted to translational motion of the atoms. Short-time dynamics on a picosecond time scale is seen in ballistic motion and vibration. Apart from some reduction in soft vibrations, not much is known about their aging effects. The longer time dynamics is most easily seen in atomic diffusion. Hórvath et al. $^{4}$ experimentally observed a decrease of the diffusion coefficients of metallic glasses by an order of magnitude during aging. The other simple class, colloidal suspensions, has been studied extensively in recent years (see, e.g., Ref. 5), but it involves Brownian dynamics which could have some influence on aging.

We use molecular dynamics simulation to study the relation between aging of the dynamics and of energy and pressure in a model metallic glass. As the dynamic variable we take the diffusivity, since we expect this to be most directly coupled to energy and volume relaxation. Our simulation is done at near zero pressure using a slightly modified version of the binary Lennard-Jones system. ${ }^{6}$ There are several earlier studies for this system, mostly with a temperature-independent density, which implies high pressures that increase with temperature. A scaling law for the aging of the intermediate scattering function was found, ${ }^{7}$ which was later disputed. ${ }^{8}$ These calculations were done for stochastic velocity distributions so that a direct comparison with the present calculation, using Newtonian dynamics, is not possible. From calculations of the change of the inherent energies it was found that a single fictive temperature $T_{f}$ suffices to describe the state above the mode coupling temperature, whereas at lower temperature a history dependence is observed. ${ }^{9}$ A decrease of the number of atomic jumps per time unit with aging time was reported. ${ }^{10}$

\section{CALCUlational DetaIls}

The present calculations are done for a binary LennardJones system described by

$$
V_{i j}(R)=4 \epsilon_{i j}\left[\left(\sigma_{i j} / R\right)^{12}-\left(\sigma_{i j} / R\right)^{6}+A_{i j} R+B_{i j}\right],
$$

where the subscripts $i$ and $j$ denote the two species $A$ and $B$. The potential cutoff was set at $R_{c}=3 \sigma$. For the parameters we took the values of Kob and Andersen: ${ }^{6} \epsilon_{A A}=\epsilon=\sigma_{A A}=\sigma=$ $1, \epsilon_{B B}=0.5, \sigma_{B B}=0.88, \epsilon_{A B}=1.5$, and $\sigma_{A B}=0.8$. The parameters $A_{i j}$ and $B_{i j}$ ensure continuity of the potential and its first derivative at the cutoff. All masses are set to $m_{j}=1$. As usual, in the following we will give all results in the reduced units of energy $\epsilon, \sigma$, and atomic mass. To compare with real metallic glasses one can equate one time unit $\left[\left(\epsilon / m \sigma^{2}\right)^{-1 / 2}\right]$ roughly to $1 \mathrm{ps}$. The time step is $\Delta t=0.005$. Control runs with $\Delta t=0.0005$ showed no significant deviation. The heat bath is simulated by comparing the temperature averaged over 20 time steps with the nominal temperature. At each time step $1 \%$ of the temperature difference is adjusted by random additions to the particle velocities. Apart from the very first steps of the aging procedure, the correction, after excursions of the temperature due to relaxations, does not exceed $10^{-4}$ of the average velocity. This procedure assures that existing correlations between the motion of atoms are only minimally affected. The calculations are done with constant volume and periodic boundary conditions. The system size was $N=5488$ with a ratio of $4: 1$ between $A$ and $B$ atoms.

For the present aging studies 64 samples for $T \geqslant 0.4$ and 8 samples for $T<0.4$ were suddenly quenched from equilibrated and, respectively, well aged samples at $T_{i}=$ $T+0.2$ to the aging temperature $T$. The densities were fixed 
to give, after aging, $p \approx 0$. The maximal final pressure was $p_{\infty}=0.03$. In units appropriate to a metallic glass, $p_{\infty}$ would be less than 1 kbar. Neither segregation nor crystallization was observed during the aging runs. In control runs with different quench protocols, including quenches by $\Delta T=0.08$ from above to below $T_{c}$, the aging rates were found to be independent of the procedure within $20 \%$. The weights of the aging terms reflect the history of the glass.

\section{RESULTS}

Earlier we reported, for this system at zero pressure, the diffusional isotope effect ${ }^{11}$ and the pressure dependence (activation volume). ${ }^{12}$ Above $T_{c} \approx 0.36$ the diffusion coefficients of both components can be given by a mode coupling theory (MCT) expression ${ }^{13} D(T)=D_{0}^{\mathrm{MCT}}\left(T-T_{c}\right)^{\gamma}$ with $T_{c}=0.36 \epsilon / k$, and below by an Arrhenius expression $D(T)=$ $D_{0}^{\text {Arrh }} \exp \left(-E_{a} / k T\right)$. From the isotope effect we conclude that, upon lowering the temperature, diffusion becomes collective and, at the critical MCT temperature $T_{c} \approx 0.36$, involves in excess of ten atoms, in agreement with the chain- (string-)like motion reported for such systems. Accordingly, the diffusional activation volumes are smaller than the atomic volume, and as expected from MCT they show a peak at $T_{c}$. At $T=$ $0.32 \approx 0.9 T_{c}$ the aging of both the diffusivity and the dynamic heterogeneity can be described by a simple exponential law. The same aging constant not only describes the relaxation of the diffusivities of both components, but also the increase of their respective non-Gaussianity parameters $\alpha_{\mathrm{NG}}$, which measure the dynamic heterogeneity. During the aging process $\alpha_{\mathrm{NG}}$ increased by about a factor of 2 and the time to reach this maximum grew simultaneously: both the strength and the lifetime of the dynamic heterogeneity increase with aging. The better aged the glass, the higher is its dynamic heterogeneity. ${ }^{14}$

In all runs pressure and energy were monitored. The timedependent diffusion constant was calculated from the slope of the atomic mean-square displacements:

$$
D(t)=\frac{1}{6} \frac{d\left\langle s^{2}(t)\right\rangle}{d t} .
$$

This expression holds only for times large enough to exclude the ballistic and vibrational regimes, and at lower temperatures also the plateau effect, which is a signature of reversible motion that is also present in equilibrium. Therefore, short-time aging effects of the diffusivity cannot be extracted using this expression.

We fit the time evolution by a KWW expression

$$
Q(T, t)=Q_{\infty}(T)+\Delta Q(T) e^{-\left[\alpha_{Q}(T) t\right]^{\beta(T)}},
$$

where $Q$ stands for pressure $(p)$, internal energy $(E)$, or the diffusion coefficients $D_{x}$ of the two components. $Q_{\infty}$ represents the long-time limit. It should be noted that the KWW expression with $\beta \neq 1$ depends on the waiting time $t_{w}$. It changes under the transformation $t=t_{w}+t^{\prime}$. For $\beta=1$ only the prefactor $\Delta Q(T)$ depends on $t_{w}$. The same holds for our result, Eq. (4). The aging of the diffusion coefficients can be fitted without a stretching factor in Eq. (3). A possible stretching effect, $\beta_{D} \neq 1$, is hidden in the inaccessible shorttime aging. We do the fitting with $\beta_{D}=1$ and a common $\alpha_{D}(T)$ for both components. The resulting long-time diffusion

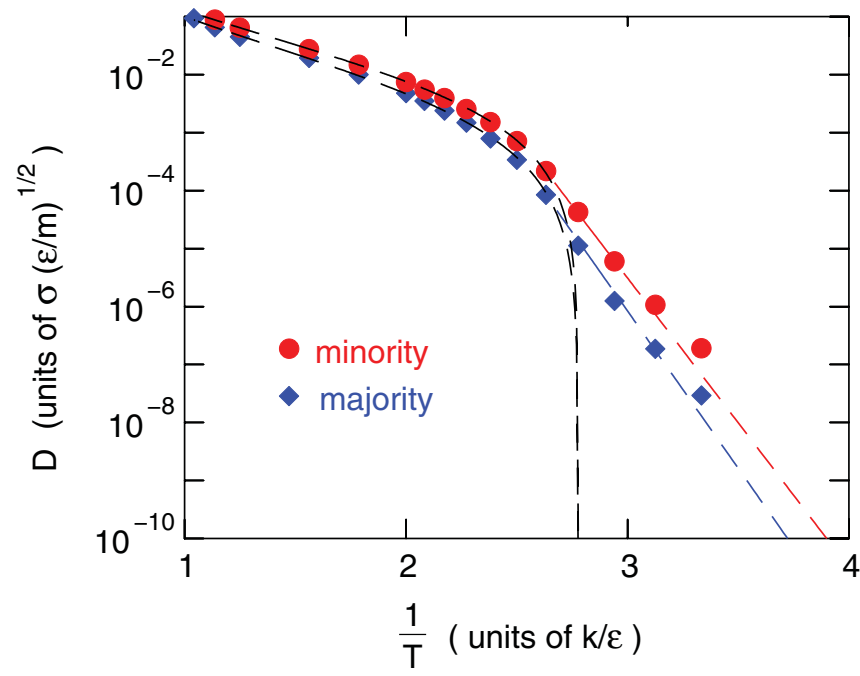

FIG. 1. (Color online) Diffusion coefficients $D_{\infty}$ (majority $A$ atoms, diamonds; minority $B$ atoms, circles) at near-zero pressure against inverse temperature (all in reduced units). The dashed lines show the fits with the MCT and Arrhenius expressions, respectively.

coefficients are shown in Fig. 1. The dashed lines show the MCT fit for high temperatures and the Arrhenius lines below $T_{c}$.

Figure 2 , top, illustrates the aging of $D$ at $T \approx 0.9 T_{c}$ [symbols denote the simulation; dashed red lines are fits with Eq. (3)]. The diffusivities drop by an order of magnitude during aging. For longer times there is a small additional decrease of the diffusivity. The diffusivity follows the slower pressure relaxation. For the present system this effect is small and only slightly affects the numerical values. The much slower

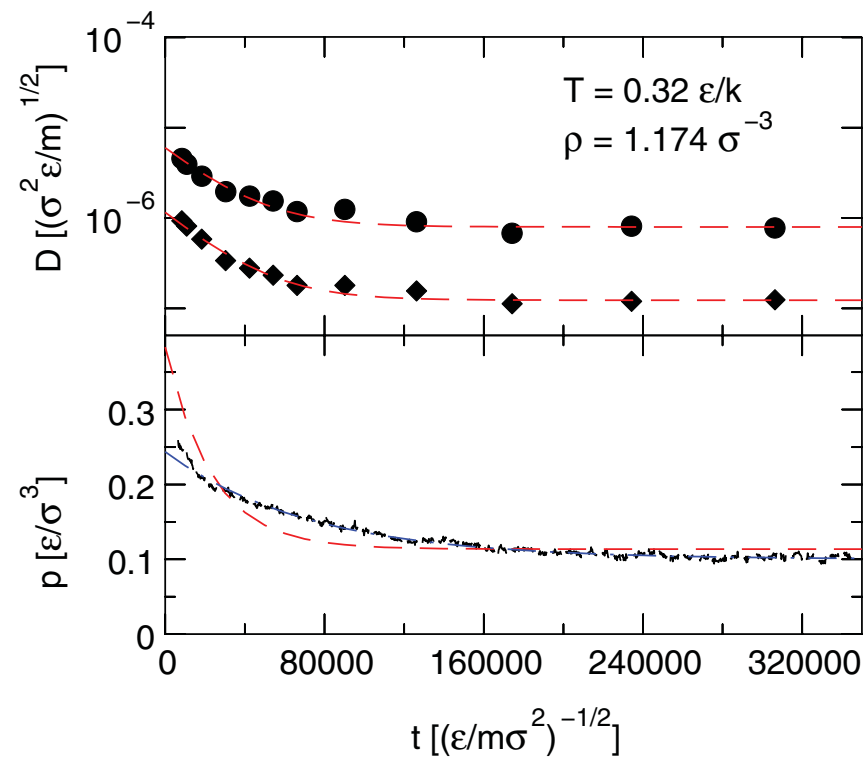

FIG. 2. (Color online) Top: diffusion coefficients (majority $A$ atoms, solid diamonds; minority $B$ atoms, solid circles) against aging time. The red dashed line is the fit using Eq. (3), with $\alpha_{D}=4.3 \times 10^{-5}$ and $\beta_{D}=1$, in LJ units. Bottom: pressure against aging time. Red dashed line: fit with $\alpha_{D}$; blue dash-dotted line: fit with $\alpha_{p}=1.4 \times 10^{-5}$ in LJ units. 


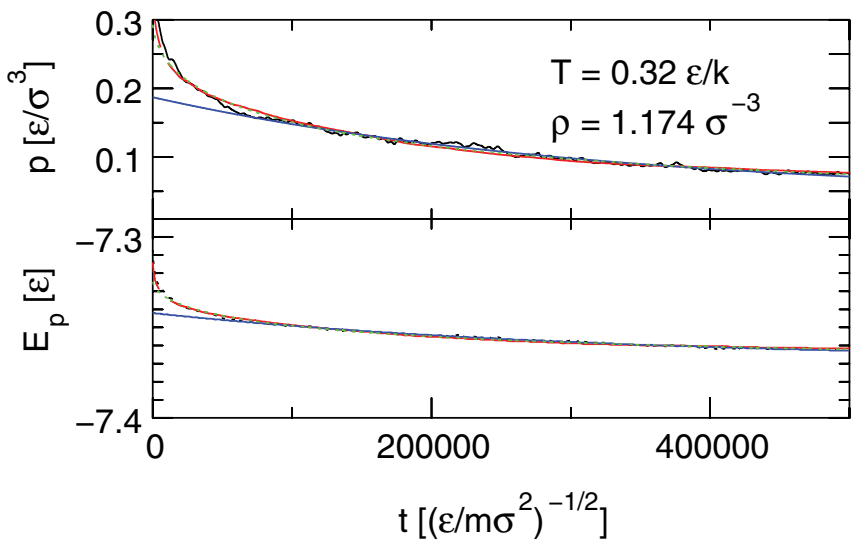

FIG. 3. (Color online) Pressure (top) and potential energy (bottom) against aging time. Blue solid line: fit according to Eq. (3) without stretching exponent; red dashed line: fit with stretching exponent $\beta=0.5$; green dash-dotted line: fit according to Eq. (4).

decrease of the pressure is depicted in Fig. 2, bottom. To illustrate the slower decay, the red dashed curve indicates a fit with the aging rate of the diffusion, with $\alpha_{p}=\alpha_{D}$, and $\beta_{p}=1$. A much better fit is obtained with $\alpha_{p} \approx \alpha_{D} / 3$. For short times the simple exponential expression, for $\beta=1$, underestimates the pressure decrease. Due to the pronounced stretching behavior (see below), this value of $\alpha_{p}$ is still too high. A fit to the pressure decay over longer time scales, Fig. 3, gives $\alpha_{p}$ reduced by a factor of 4 .

As reported earlier for the simultaneous aging of diffusivity and dynamic heterogeneity, ${ }^{14}$ we find that pressure and internal energy age in parallel; see Fig. 3. For long times aging can be fitted by an exponential law with $\alpha_{p}=\alpha_{E}$ (solid blue line). To fit the aging also at shorter times, stretching has to be included. An accurate determination of $\beta$ with sufficient accuracy is not possible from the present data. A value of $\beta_{p}=\beta_{e}=0.5$ gives an excellent fit. This value is roughly compatible with the ones obtained from experiment. ${ }^{1}$

The decay constants for diffusivity $\alpha_{D}$ and for pressure and energy $\alpha_{p}=\alpha_{e}$ are plotted in Fig. 4 against inverse temperature. Above $T_{c}$ diffusivity and pressure age with the same constants and no stretching is discernible. Below $T_{c}$ the behavior changes. The two aging curves split; aging of the diffusivity is much faster than the one of pressure and energy. Apart from the initial stage an exponential law suffices to fit the aging of $D$. The much weaker additional aging due to the aging of pressure is neglected. In contrast, the aging curves for both energy and pressure show a pronounced stretched exponential (KWW) shape. The solid symbols show the exponential fit to the long-time decay and the open symbols the KWW fit with $\beta=0.5$. Over the whole temperature range the aging rate of pressure and energy follows approximately the diffusivity, $\alpha_{p}=\alpha_{E} \approx 24 D_{\text {eff }} / \sigma^{2}$ (dashed lines), with $D_{\text {eff }}^{-1}=0.8 D_{A}^{-1}+0.2 D_{B}^{-1}$. Remarkably this holds both in the MCT and Arrhenius regimes.

This suggests introducing an aging length $\ell_{p, E}$ to describe aging of $p$ and $E$ :

$$
p(T, t)=p_{\infty}(T)+\Delta p e^{-\left(\left\langle s_{\text {eff }}^{2}(t)\right\rangle-\left\langle s_{\text {ball }}^{2}\right\rangle\right) / \ell_{p, E}(T)} .
$$

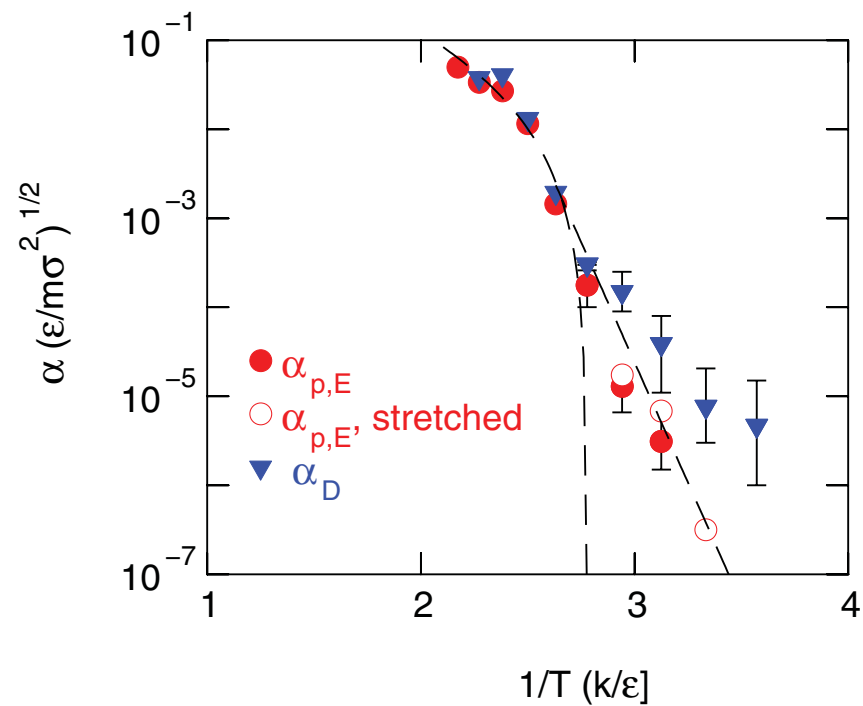

FIG. 4. (Color online) Aging exponents for pressure and internal energy (circles) and for the diffusion coefficient and the dynamic heterogeneity (triangles). The solid symbols refer to fits without stretching exponent, the open symbols to a stretching exponent $\beta=0.5$. The error bars are estimates of the fit reliability. The dashed lines show the fits with the MCT and Arrhenius expressions for $D_{\infty}$ above and below $T_{c}$, respectively.

Here $s_{\text {ball }}$ is the ballistic (vibrational) mean-square displacement, and the effective mean square displacement is defined by $1 /\left\langle s_{\text {eff }}^{2}\right\rangle=0.8 /\left\langle s_{A}^{2}\right\rangle+0.2 /\left\langle s_{B}^{2}\right\rangle$. In the present case $\left\langle s_{\text {eff }}^{2}\right\rangle \approx$ $\left\langle s_{A}^{2}\right\rangle$. As shown by the green dash-dotted line in Fig. 3, Eq. (4) gives a near perfect fit of the aging of pressure and energy, including the stretching effect. The curves with the KWW fit nearly coincide with the fit by Eq. (4). The observed stretching in the aging of pressure and energy is thus an effect of the faster aging of the diffusivity. Since there is little change in the compressibility during aging, this holds for the aging of volume for fixed pressure.

In Fig. 4 it was shown that $\alpha_{p, E} \approx 24 D_{\text {eff }} / \sigma^{2}$, which corresponds to $\ell_{p, E} \approx 0.5 \sigma$. In Ref. 12 the change of diffusion coefficient with pressure was studied for the equilibrium or long-time limit. In this work a sharp maximum at $T=T_{c}$ was observed in the activation volume

$$
V_{\text {act }}(T)=-k T\left[\frac{\partial \ln D(T)}{\partial p}\right]_{T} .
$$

$V_{\text {act }}$ is a measure of the coupling between diffusivity and pressure. Since Eq. (4) couples the diffusivity and the change of pressure during aging, a similar temperature dependence of $\ell_{p, E}$ could be expected. Figure 5 shows both $\ell_{p, E}(T)$ (red solid circles) and $V_{\text {act }}$ (blue diamonds) against temperature. Whereas $\ell_{p, E}(T)$ roughly has the the expected value of $0.5 \sigma$, it peaks near $T_{c}$ as previously observed for the equilibrium diffusional activation volume. The two peak positions seem not to fully coincide. This is most probably an effect of the aging history. The calculation of the activation volume was as near as possible under equilibrium conditions. During the aging calculation the pressure changed with time, and the critical MCT temperature of a freshly quenched glass is not well defined. $\left\langle s_{\text {eff }}^{2}(t)\right\rangle$ is an integral over stages with different pressures and $T_{c}$. We would 


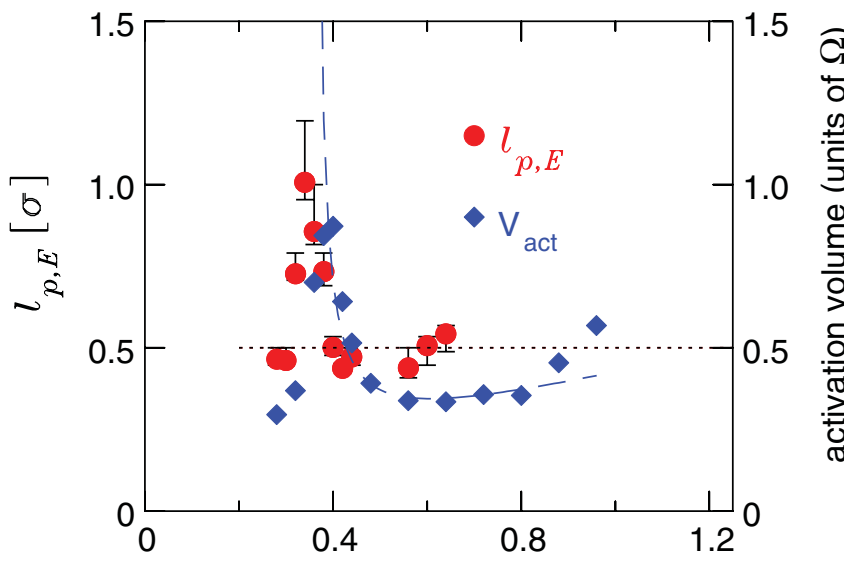

$\mathrm{T}[\varepsilon / \mathrm{k}]$

FIG. 5. (Color online) Red solid circles: aging length against temperature. The dotted line represent the approximate fit with the MCT and Arrhenius expressions in Fig. 4. Blue solid diamonds and dashed line: calculated diffusional activation volume and MCT fit. ${ }^{12}$ The error bars are estimates of the fit reliability.

expect that, starting from a glass nearer to its equilibrium, the two maxima will be closer in temperature.

\section{DISCUSSION}

If one considers aging as an evolution of the glass to a lower-energy state, one can envisage the aging process as the annihilation of defects, whatever they might be. $\Delta Q(T)$ in Eq. (3) would then be the concentration of such defects at the start, $c_{\text {def }}(0)$, times the defect strength. ${ }^{14}$ The exponential gives the annihilation rate, and a stretching in the KWW law could be ascribed to a distribution of activation energies. ${ }^{1}$ With one type of defect only, all quantities age in parallel, only distinguished by different defect strengths. Such a scenario is compatible with our results for $T>T_{c}$. However, at lower temperatures we find at least two different aging rates in the system: a higher rate for the diffusivity and a much lower one for pressure and energy.

It has been known for a long time that near $T_{c}$ a transition from homogeneous to heterogeneous dynamics occurs. One standard measure of heterogeneity is the so called nonGaussianity parameter (NGP), ${ }^{15} \alpha_{2}$. It is a measure of the deviation of the van Hove autocorrelation function from a Gaussian shape, as one would have for isotropic random motion. Such deviations are regularly observed in simulations of metallic systems; see, e.g., Ref. 16. Upon cooling the high pressure LJ system to its $T_{c}$, a maximal value of the NGP, $\alpha_{\max } \approx 2$, was observed. That is ten times the value at $T=2 T_{c}{ }^{6}{ }^{6}$ Due to the high pressure in that simulation, the $T_{c}$ was about $20 \%$ higher than the present value. For the present system under near-zero pressure conditions, a similar value at $T_{c}$ was reported. Upon cooling beyond a temperature somewhat above $T_{c}, \alpha_{\max }$ started to increase rapidly. ${ }^{14}$

Taking the picture of an inherent energy landscape, the system moves from one local minimum to another by a collective motion of a few atoms. ${ }^{17}$ Lowering the temperature, the possible paths for such transitions are thinned out. In simulations of metallic systems this collective motion was seen to be chain- or string-like. In the glassy state diffusional motion was reported to involve such chain- (string-)like structures,${ }^{18}$ closely correlated to the quasilocalized vibrations that are thought to cause the "boson peak" in the inelastic scattering intensity. ${ }^{19,20}$ Similar structures were seen in the undercooled melt. ${ }^{21,22}$ If such a string of atoms has jumped once, it is more likely to jump again, thus creating spikes of activity. ${ }^{16,20,23}$ After each jump some atoms will leave the string and others join. This picture provides an explanation of the time evolution of the NGP and its increase upon lowering the temperature. ${ }^{24}$ Similar behavior is observed for different systems. ${ }^{24}$ Monitoring the tails of the van Hove function, a general scenario was found for systems ranging from silica to colloids and grains, and again was rationalized with a model of correlated jumps. ${ }^{25}$

This scenario is held to be responsible for the breakdown of the Stokes-Einstein relation (SER) between diffusivity and viscosity. ${ }^{26}$ Diffusivity is determined mainly by the fast atoms, while viscosity necessitates the slow atoms to move. Similarly pressure and energy relaxation will involve at longer times all atoms. Recent experiments on $\mathrm{Ni}_{3} 6 \mathrm{Zr}_{6} 4$ have shown that the SER breaks down far above $T_{c} .{ }^{27}$ The temperature dependence of the ratio between viscosity and diffusion points to a collective mechanism. The same break in the temperature dependence in the SER was found in a computer simulation of $\mathrm{CuZr}_{2} \cdot{ }^{28}$

The mean-field description of mode coupling theory predicts a freezing of the system at $T_{c}$. However, before this happens dynamic processes take over that are not included or are added to the simple theory as hopping processes take over. For diffusion this can be seen in Fig. 1 as the crossover to an Arrhenius behavior above $T_{c}$. For different properties the weighting between the different dynamic processes will differ, and thus there will not be an exact universal crossover temperature. The onset of the freezing according to mode coupling theory can, however, be seen in the pressure dependence of the diffusion ${ }^{12}$ and in the present work in the spike in the aging length; see Fig. 5.

The crossover from a homogeneous scenario, with a single aging rate above $T_{x} \approx T_{c}$, to at heterogeneous one, with at least two different rates below $T_{x}$, implies that a single fictive temperature is not sufficient to describe the state of the system below $T_{x}$. This is in agreement with earlier work., ${ }^{2,9,29}$

Molecular dynamics is limited to small system sizes and times of up to a few microseconds. Therefore, no statement about possible additional ultraslow relaxation processe $\mathrm{s}^{30} \mathrm{can}$ be made. We are also limited to temperatures not too far below $T_{c}$ and therefore to not-too-high dynamic heterogeneities. At lower temperatures the averaging used for $\left\langle s_{\text {eff }}^{2}(t)\right\rangle$ might be to simple. Inverse averaging would put a higher weight on the slow particles. Aging experiments measuring simultaneously volume and diffusivity in metallic glasses could clarify this.

\section{CONCLUSION}

In conclusion, we studied aging in a binary model glass near zero pressure. In agreement with a homogeneous scenario, above a temperature $T_{x} \approx T_{c}$ energy, pressure, and diffusivity 
age with the same rate nearly exponentially. Below $T_{x}$ the diffusivity ages much faster than energy and pressure. This crossover coincides with the onset of a marked dynamic heterogeneity. A pronounced stretching is seen in the aging of energy and pressure. The decrease of their aging rates upon cooling roughly follows the drop of the diffusivity, both in the homogeneous (MCT) regime and in the low-temperature
Arrhenius one. Aging of energy and pressure can be expressed in terms of the mean-square displacement. Their aging can be expressed in terms of an aging length that spikes similarly to the pressure dependence of the diffusivity. The stretched exponential aging of energy and pressure results from the faster aging of the diffusivity, which relates it to the dynamic heterogeneity.
${ }^{1}$ I. M. Hodge, J. Non-Cryst. Solids 169, 211 (1994).

${ }^{2}$ R. L. Leheny and S. R. Nagel, Phys. Rev. B 57, 5154 (1998).

${ }^{3}$ P. Lunkenheimer, R. Wehn, U. Schneider, and A. Loidl, Phys. Rev. Lett. 95, 055702 (2005).

${ }^{4}$ J. Horvath, K. Pfahler, W. Ulfert, W. Frank, and H. Kronmüller, Mater. Sci. Forum 15-18, 523 (1987).

${ }^{5}$ P. Yunker, Z. Zhang, K. B. Aptowicz, and A. G. Yodh, Phys. Rev. Lett. 103, 115701 (2009).

${ }^{6}$ W. Kob and H. C. Andersen, Phys. Rev. E 51, 4626 (1995).

${ }^{7}$ W. Kob and J. L. Barrat, Phys. Rev. Lett. 78, 4581 (1997).

${ }^{8}$ U. Müssel and H. Rieger, Phys. Rev. Lett. 81, 930 (1998).

${ }^{9}$ I. Saika-Voivod and F. Sciortino, Phys. Rev. E 70, 041202 (2004).

${ }^{10}$ K. Vollmayr-Leem, J. Chem. Phys. 121, 4781 (2004).

${ }^{11}$ H. R. Schober, Solid State Commun. 119, 73 (2001).

${ }^{12}$ H. R. Schober, Phys. Rev. Lett. 88, 145901 (2002).

${ }^{13}$ W. Götze and A. Sjölander, Rep. Prog. Phys. 55, 241 (1992).

${ }^{14}$ H. R. Schober, Phys. Chem. Chem. Phys. 6, 3654 (2004).

${ }^{15}$ A. Rahman, Phys. Rev. 136, A405 (1964).

${ }^{16}$ F. Faupel, W. Frank, M.-P. Macht, V. Naundorf, K. Rätzke, H. R. Schober, S. K. Sharma, and H. Teichler, Rev. Mod. Phys. 75, 237 (2003).

${ }^{17}$ T. B. Schrøder, S. Sastry, J. C. Dyre, and S. C. Glotzer, J. Chem. Phys. 112, 9834 (2000).
${ }^{18}$ H. R. Schober, C. Oligschleger, and B. B. Laird, J. Non-Cryst. Solids 156, 965 (1993).

${ }^{19}$ B. B. Laird and H. R. Schober, Phys. Rev. Lett. 66, 636 (1991); H. R. Schober and B. B. Laird, Phys. Rev. B 44, 6746 (1991).

${ }^{20} \mathrm{C}$. Oligschleger and H. R. Schober, Phys. Rev. B 59, 811 (1999).

${ }^{21}$ H. R. Schober, C. Gaukel, and C. Oligschleger, Prog. Theor. Phys. Suppl. 126, 67 (1997).

${ }^{22}$ C. Donati, J. F. Douglas, W. Kob, S. J. Plimpton, P. H. Poole, and S. C. Glotzer, Phys. Rev. Lett. 80, 2338 (1998).

${ }^{23}$ H. Teichler, Defect Diffus. Forum 143-147, 717 (1997).

${ }^{24}$ D. Caprion, J. Matsui, and H. R. Schober, Phys. Rev. Lett. 85, 4293 (2000).

${ }^{25}$ P. Chaudhuri, L. Berthier, and W. Kob, Phys. Rev. Lett. 99, 060604 (2007).

${ }^{26}$ V. Zöllmer, K. Rätzke, F. Faupel, and A. Meyer, Phys. Rev. Lett. 90, 195502 (2003).

${ }^{27}$ J. Brillo, A. I. Pommrich, and A. Meyer, Phys. Rev. Lett. 107, 165902 (2011)

${ }^{28}$ X. J. Han and H. R. Schober, Phys. Rev. B 83, 224201 (2011).

${ }^{29}$ R. Richert, Phys. Rev. Lett. 104, 085702 (2010).

${ }^{30}$ R. S. Miller and R. A. MacPhail, J. Chem. Phys. 106, 3393 (1997). 